\title{
The Lie Derivative, Invariance Conditions, and Physical Laws
}

\author{
W.-H. Steeb \\ Gesamthochschule Paderborn, Arbeitsgruppe Theoretische Physik, Paderborn
}

Z. Naturforsch. 33a, 742-748 (1978); received March 4, 1978

The properties of the Lie derivative of a differential form with respect to a vector field are applied to some physical problems. In particular we study balance equations.

\section{Introduction}

The concept of the Lie derivative of a differential form (or a vector field) with respect to a vector field plays an important role in many domains in physics. Applications have been made to classical mechanics, hydrodynamics, optics, quantum mechanics, supergravity, theory of relativity and statistical mechanics [1-22]. Moreover partial differential equations (linear and non-linear), which play an important role in physics, can be treated within this so-called geometric approach $[23,24]$.

In the present paper we show how some physical laws can be derived within this approach.

In Section 2 we give a summary of the rules for the Lie derivative and the related operations, namely inner product, exterior derivative, co-derivative, and star operation. Some additional rules to those found in the literature will be given $[1-6$, $23,25]$. Section 3 is devoted to introducing the notion of invariance of a differential form with respect to a vector field. Moreover, we derive some relations for forms which are invariant with respect to a vector field. Several examples which are important in physics will be given (Section 4). In particular, we study continuity equations.

\section{Mathematical Preliminaries}

Let $M$ be an $n$-dimensional $C^{\infty}$-differentiable manifold with local coordinates $x_{i}$. Real valued $C^{\infty}$-vector fields (denoted by $V, W, X, X_{1}, \ldots, X_{n}$, $Y, Z$ ) and real valued $C^{\infty}$-differential forms (denoted by Greek letters) on $M$ can be considered. The components of the vector field $X$ are denoted by $x^{i} \partial / \partial x_{i}$, this means $X=X^{1} \partial / \partial x_{1}+\cdots+X^{n} \partial / \partial x_{n}$ (in local coordinates). Moreover, we have the space of the real valued $C^{\infty}$-functions $f: M \rightarrow R$. We assume throughout that all mathematical objects are smooth.

Now the concept of the Lie derivative of a form w.r.t. a vector field can be introduced $[1-6]$. The
Lie derivative of a form $\alpha$ w.r.t. $X$ is defined by the derivative of $\alpha$ along the integral curve $t \rightarrow \Phi_{t}$ of $X$, i.e.,

$$
L_{X} \alpha=\lim _{t \rightarrow 0}\left\{\left(\Phi_{t} * \alpha-\alpha\right) / t\right\} .
$$

The definition of the Lie derivative is not useful for practical calculations. Rather, we use the important identity

$$
\left.\left.L_{X} \alpha=\mathrm{d}(X\lrcorner \alpha\right)+X\right\lrcorner(\mathrm{d} \alpha),
$$

$\mathrm{d} \alpha$ denotes the exterior derivative of the form $\alpha$. $X\lrcorner \alpha$ is the contraction of $\alpha$ by $X$ (also called inner product). For a form, $\alpha$, the Lie derivative $L_{X}$ may be viewed as the propagator of the form, $\alpha$, down the trajectories of the vector field $X$.

For vector fields the Lie bracket can also be considered as a Lie derivative. This means

$$
\left(L_{X} Y\right) f=[X, Y] f=X(Y f)-Y(X f) .
$$

Before introducing the notion of invariance we give the rules of manipulation. Most of the rules can be found in the literature $[1-6],[23,25]$. $\alpha$ and $\beta$ are $r$ - and $s$-forms respectively. $f, f_{1}, \ldots, f_{4}$, $g$ are arbitrary functions. $c, c_{1}, \ldots, c_{4}$ denote constants. The Lie derivative of a differential form w.r.t. a vector field has the following properties:

$$
\begin{aligned}
& L_{X}\left(c_{1} \alpha+c_{2} \beta\right)=c_{1} L_{X} \alpha+c_{2} L_{X} \beta, \\
& L_{\left(c_{1} X+c_{2} Y\right)} \alpha=c_{1} L_{X} \alpha+c_{2} L_{Y} \alpha, \\
& L_{X}(\alpha \wedge \beta)=\left(L_{X} \alpha\right) \wedge \beta+\alpha \wedge\left(L_{X} \beta\right), \\
& L_{f X} \alpha=f\left(L_{X} \alpha\right)+\mathrm{d} f \wedge(X \vdash \alpha), \\
& \mathrm{d}\left(L_{X} \alpha\right)=L_{X}(\mathrm{~d} \alpha), \\
& \left.\left.\left.L_{X}(Y\lrcorner \alpha\right)=[X, Y]\right\lrcorner \alpha+Y\right\lrcorner\left(L_{X} \alpha\right), \\
& L_{[X, Y]} \alpha=L_{X}\left(L_{Y} \alpha\right)-L_{Y}\left(L_{X} \alpha\right), \\
& \left.X\lrcorner\left(L_{X} \alpha\right)=L_{X}(X\lrcorner \alpha\right), \\
& L_{X} f \alpha=(X f) \alpha+f\left(L_{X} \alpha\right), \\
& L_{X} f g=f\left(L_{X} g\right)+g\left(L_{X} f\right), \\
& L_{X} c=0 .
\end{aligned}
$$


In addition we have the rules for the exterior derivative, the transformation properties of differential forms (where $\Phi: M \rightarrow N: p \rightarrow \Phi(p)$ is a differentiable transformation from the manifold $M$ into the manifold $N$ ), the inner product (which is a purely algebraic bilinear operation), and the Lie bracket of vector fields.

$$
\begin{aligned}
& \left(f_{1} \alpha+f_{2} \beta\right) \wedge\left(f_{3} \gamma+f_{4} \delta\right) \\
& =f_{1} f_{3}(\alpha \wedge \gamma)+f_{1} f_{4}(\alpha \wedge \delta) \\
& +f_{2} f_{3}(\beta \wedge \gamma)+f_{2} f_{4}(\beta \wedge \delta), \\
& \alpha \wedge \beta=(-1)^{r s} \beta \wedge \alpha, \\
& \alpha \wedge(\beta \wedge \gamma)=(\alpha \wedge \beta) \wedge \gamma, \\
& \mathrm{d}\left(c_{1} \alpha+c_{2} \beta\right)=c_{1} \mathrm{~d} \alpha+c_{2} \mathrm{~d} \beta, \\
& \mathrm{d}(\alpha \wedge \beta)=(\mathrm{d} \alpha) \wedge \beta+(-1)^{r} \alpha \wedge(\mathrm{d} \beta), \\
& \mathrm{d} c=0, \\
& \operatorname{dd} \alpha=0 \text {, } \\
& \mathrm{d} f=\sum_{i=1}^{n} \frac{\partial f_{i}}{\partial x_{i}} \mathrm{~d} x_{i}, \\
& \Phi^{*}(\alpha+\beta)=\Phi^{*} \alpha+\Phi^{*} \beta, \\
& \Phi^{*}(\alpha \wedge \beta)=\left(\Phi^{*} \alpha\right) \wedge\left(\Phi^{*} \beta\right), \\
& \Phi^{*}(\mathrm{~d} \alpha)=\mathrm{d}\left(\Phi^{*} \alpha\right) \text {, } \\
& \Phi^{*}(\mathrm{~d} f)=\mathrm{d}(f \circ \Phi), \\
& \Phi^{*}(f \alpha)=(f \circ \Phi) \Phi^{*} \alpha, \\
& \left.\left.\left(f_{1} X\right\lrcorner+f_{2} Y\right\lrcorner\right)\left(f_{3} \alpha+f_{4} \beta\right) \\
& \left.\left.=f_{1} f_{3}(X\lrcorner \alpha\right)+f_{1} f_{4}(X\lrcorner \beta\right) \\
& +f_{2} f_{3}(Y-\alpha)+f_{2} f_{4}(Y-\beta) \text {, } \\
& X\lrcorner f=0 \text {, } \\
& X\lrcorner X=0 \text {, } \\
& X\lrcorner(X\lrcorner \alpha)=(X\lrcorner X)-\alpha=0, \\
& X\lrcorner(\alpha \wedge \beta)=(X\lrcorner \alpha) \wedge \beta \\
& +(-1)^{r} \alpha \wedge(X-\beta), \\
& {\left[c_{1} X+c_{2} Y, c_{3} V+c_{4} W\right]} \\
& =c_{1} c_{3}[X, V]+c_{1} c_{4}[X, W] \\
& +c_{2} c_{3}[Y, V]+c_{2} c_{4}[Y, W],
\end{aligned}
$$

$[X,[Y, Z]]+[Z,[X, Y]]+[Y,[Z, X]]=0$.
Observe that the operators $\mathrm{d}($.$) and X\lrcorner$ (.) do not commute in general. The same is true for the operators $X\lrcorner\left(\right.$.) and $L_{Y}($.$) . However, if X=Y$, then $L_{Y}($.$\left.) and X\right\lrcorner$ (.) do commute [rule (11)]. We mention that $\operatorname{dd}()=$.0 and $X\lrcorner(X\lrcorner().)=0$, whereas $L_{X}\left(L_{X}().\right) \neq 0$. Finally, let $r \geqq 2$, then $X\lrcorner(Y\lrcorner \alpha)=-Y\lrcorner(X \alpha)$.

The linear operators $\mathrm{d}(), X\lrcorner.($.$) , and L_{X}($.$) are$ coordinate-free operators.

In general the physicist is familiar with exterior derivatives and exterior products. To calculate the inner product of a form and a vector field, we use the basic property (in local coordinates)

$$
\left.\frac{\partial}{\partial x_{i}}\right\lrcorner \mathrm{d} x_{j}=\delta_{i j}
$$

and the rules (28) through (32). The given property means that the one-forms $\left\{\mathrm{d} x_{j}, j=1, \ldots, n\right\}$ are defined to be the duals to the elements of the basis $\left\{\partial / \partial x_{i}, i=1, \ldots, n\right\}$.

For example, the contraction of a vector field with a two form plays an important role in classical mechanics. Let $X=X^{1} \partial / \partial x_{1}+\cdots+X^{n} \partial / \partial x_{n}$ and $\alpha=\mathrm{d} x_{i} \wedge \mathrm{d} x_{j}$, then

$$
\begin{aligned}
X\lrcorner\left(\mathrm{d} x_{i} \wedge \mathrm{d} x_{j}\right) & \left.\left.=(X\lrcorner \mathrm{d} x_{i}\right) \wedge \mathrm{d} x_{j}-\mathrm{d} x_{i} \wedge(X\lrcorner \mathrm{d} x_{j}\right) \\
& =X^{i} \mathrm{~d} x_{j}-X_{j} \mathrm{~d} x_{i}
\end{aligned}
$$

where $\beta \wedge f=f \wedge \beta=f \beta$. As a further example, let $M=\mathbb{R}^{n}$ (or any open subset of $\mathbb{R}^{n}$ ) and

$$
\omega=\mathrm{d} x_{1} \wedge \cdots \wedge \mathrm{d} x_{n} .
$$

Then we have the local representation

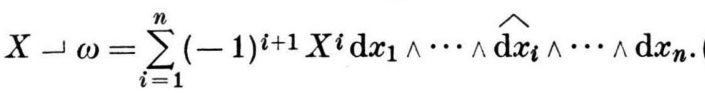

The circumflex indicates omission. Notice that $(n-1)$-forms and vector fields are in one-to-one correspondence via $X \mapsto X\lrcorner \omega$.

To sum up, the rules [Eqs. (4) through (14), and Eqs. (32), (37)] enables us to calculate, in local coordinates, the Lie derivative of an arbitrary form w.r.t. an arbitrary vector field.

The notion of the co-derivative $\delta \alpha$ of a form $\alpha$ is also important in physics. It is defined (in euclidean coordinates, $M=$ Riemannian manifold) for an $r$-form $(r \leqq n) \alpha=f(x) \mathrm{d} x_{1} \wedge \cdots \wedge \mathrm{d} x_{r}$ as

$\delta \alpha=\sum_{i=1}^{n}(-1)^{i+1} \frac{\partial f}{\partial x_{i}} \widehat{\mathrm{d} x_{1}} \wedge \cdots \wedge \mathrm{d} x_{i} \wedge \cdots \wedge \mathrm{d} x_{r}$

and $\delta f=0 . \delta($.$) is a linear operator. Observe that$ in contrast to the differential operator $d$, the co- 
differential operator $\delta$ involves the metric structure of $M$ (Riemannian manifold) in an essential way. Let $\omega=f(x) \mathrm{d} x_{1} \wedge \cdots \wedge \mathrm{d} x_{n}$, then $(\mathrm{d} \delta+\delta \mathrm{d}) \omega=$ $(\Delta f) \mathrm{d} x_{1} \wedge \cdots \wedge \mathrm{d} x_{n}$, where $\Delta$ is the Laplace operator, i.e. $\Delta=\partial^{2} / \partial x_{1}{ }^{2}+\cdots+\partial^{2} \partial x_{n}{ }^{2}$. Note that $\delta \delta \alpha=0$ [analogously to $\mathrm{dd} \alpha=0$ and $X\lrcorner(X\lrcorner \alpha)=0$ ]. On the other hand $\delta L_{X} \alpha \neq L_{X} \delta \alpha$, in contrast to rule (8).

The star operator $*($.$) , also important in physics,$ is defined for an $r$-form $\alpha=\mathrm{d} x_{i_{1}} \wedge \cdots \wedge \mathrm{d} x_{i_{r}}$ as $(M$ orientable manifold)

$$
* \alpha= \pm \mathrm{d} x^{j}{ }_{1} \wedge \cdots \wedge \mathrm{d} x_{j_{n-r}},
$$

where $\left(i_{1}, \ldots, i^{r}, j_{1}, \ldots, j_{n-r}\right)$ is a permutation of $(1, \ldots, n)$ and \pm is the sign of the permutation. $*($.$) is a linear purely algebraic operator which$ obeys the rule $*(f \alpha+g \beta)=f(* \alpha)+g(* \beta) . \alpha \rightarrow * \alpha$ defines an isomorphism. As an example, let $\omega=\mathrm{d} x_{1} \wedge \cdots \wedge \mathrm{d} x_{n}\left(M=\mathbb{R}^{n}\right)$, then $* \omega=1$. In $\mathbb{R}^{2}$, with the rectangular coordinates $\left(x_{1}, x_{2}\right)$, we have $* \mathrm{~d} x_{1}=\mathrm{d} x_{2}, * \mathrm{~d} x_{2}=-\mathrm{d} x_{1}$. The star operator plays an important role in the algebraic topological interpretation for basic equations in electromagnetic fields.

The operator $L_{X}($.$) can also be applied to$ covariant and contravariant tensors. The differential forms are a special class of covariant tensors. However, some physical quantities, for example the energy-momentum tensor, cannot be expressed as a differential form. Hence, for the sake of completeness we consider the Lie derivative of covariant tensors. Let $S$ and $T$ be covariant tensors. We have the following rules. Again, the operator $L_{X}($.$) is linear. This means$

$$
\begin{aligned}
L_{X}(T+S) & =L_{X} T+L_{X} S, \\
L_{X+Y} S & =L_{X} S+L_{Y} S .
\end{aligned}
$$

In analogy to Eqs. (6) and (12), one has

$$
\begin{aligned}
L_{X}(T \otimes S) & =\left(L_{X} T\right) \otimes S+T \otimes\left(L_{X} S\right),( \\
L_{X}(f T) & =(X f) T+f\left(L_{X} T\right) .
\end{aligned}
$$

In local coordinates a covariant tensor can be expressed as

$$
T=\sum_{i_{1} \ldots i_{p}=1}^{n} T_{i_{1} \ldots i_{p}} \mathrm{~d} x_{i_{1}} \otimes \cdots \otimes \mathrm{d} x_{i_{p}} .
$$

Consequently, to calculate the Lie derivative of $T$ with respect to $X$ it remains, after applying the rules given above, to determine the expression $L_{X} \mathrm{~d} x_{i}$. This can be done by making use of the rule (2). Because of $\mathrm{dd} x_{i}=0$, we obtain $L_{X} \mathrm{~d} x_{i}=$ $\left.\mathrm{d}(X\lrcorner \mathrm{d} x_{i}\right)=\mathrm{d} X^{i}$, where $\mathrm{d} X^{i}$ is given by Equation (22). With the rules given above we are able to treat invariance problems which arise in the theory of relativity $[20,21]$. Requiring that $L_{X} T=0$, then we get the so-called Killing vector fields.

\section{Invariance Conditions}

Starting with the definition given above we are able to establish the notion of invariance. Let $X$ be a vector field on $M$ and $\alpha$ a form on $M$. The requirement that a differential form, $\alpha$, be a conformal invariant w.r.t. a vector field, $X$, is realized by the statement

$$
L_{X} \alpha=g \alpha,
$$

where $g \in C^{\infty}(M)$. If $g=0$, then we call $\alpha$ invariant w.r.t. $X$. Thus we have the condition

$$
L_{X} \alpha=0 \text {. }
$$

The object $\alpha$ does not change as it propagates down the trajectories of $X$. For short we call $\alpha X$-invariant [26]. As a first example, let

$$
X=y \partial / \partial x-x \partial / \partial y
$$

and

$$
\alpha=(x f(r)-y g(r)) \mathrm{d} y-(y f(r)+x g(r)) \mathrm{d} x,
$$

where $r^{2}=x^{2}+y^{2}$. Then $L_{X} \alpha=0$. As a second example, let us consider the Lorentz group. This group is associated with the following vector fields ( $c t \rightarrow t, c=$ speed of light)

$$
\begin{aligned}
& X_{1}=t \frac{\partial}{\partial x}+x \frac{\partial}{\partial t}, \quad X_{2}=t \frac{\partial}{\partial y}+y \frac{\partial}{\partial t}, \\
& X_{3}=t \frac{\partial}{\partial z}+z \frac{\partial}{\partial t}, \quad X_{4}=y \frac{\partial}{\partial x}-x \frac{\partial}{\partial y}, \\
& X_{5}=z \frac{\partial}{\partial y}-y \frac{\partial}{\partial z}, \quad X_{6}=x \frac{\partial}{\partial z}-z \frac{\partial}{\partial x} .
\end{aligned}
$$

The quadratic form $x^{2}+y^{2}+z^{2}-t^{2}$ is invariant under the above vector fields $X_{1}, \ldots, X_{6}$. This means

$$
\begin{aligned}
& L_{X_{i}}\left(x^{2}+y^{2}+z^{2}-t^{2}\right) \\
& \quad=X_{i}\left(x^{2}+y^{2}+z^{2}-t^{2}\right)=0 .
\end{aligned}
$$

As a third example we consider the forms

$$
\begin{aligned}
\alpha & =\mathrm{d} \Phi-u \mathrm{~d} t-v \mathrm{~d} x, \\
\mathrm{~d} \alpha & =-\mathrm{d} u \wedge \mathrm{d} t-\mathrm{d} v \wedge \mathrm{d} x, \\
\beta & =\mathrm{d} u \wedge \mathrm{d} x+\mathrm{d} v \wedge \mathrm{d} t-m^{2} \mathrm{~d} x \wedge \mathrm{d} t,
\end{aligned}
$$


and the vector field

$$
X=x \frac{\partial}{\partial t}+t \frac{\partial}{\partial x}-v \frac{\partial}{\partial u}-u \frac{\partial}{\partial v},
$$

where $m^{2}$ is a constant. We obtain $L_{X} \alpha=0$ and $L_{X} \beta=0$. Since $L_{X} \alpha=0$, we find that $L_{X} \mathrm{~d} \alpha=0$ (rule (8)). Thus the forms $\alpha, \mathrm{d} \alpha$, and $\beta$ are $X$. invariant. The example has physical meaning. It describes that the (one dimensional) Klein-Gordon equation $\left(\partial^{2} \Phi / \partial t^{2}-\partial^{2} \Phi / \partial x^{2}+m^{2} \Phi=0\right)$ is invariant under the Lorentz transformation. Within the geometric approach of partial differential equations $[23,24]$ we cast the pde into an equivalent set of forms. In the present case we have put $\partial \Phi / \partial t=u$ and $\partial \Phi / \partial x=v$. The (once-extended) vector field $X$ (Eq. (52)) is associated with the Lorentz transformation.

An $X$-invariant form $\alpha$ is said to be an absolute integral invariant [26] of $X$ if in addition $X\lrcorner \alpha=0$ or equivalently if simultaneously

$$
X\lrcorner \alpha=0 \text { and } X\lrcorner \mathrm{d} \alpha=0 .
$$

A first integral of $X$ is an absolute integral invariant of order 0 .

A form $\alpha$ is said to be relative integral [26] of $X$ if $\mathrm{d} \alpha$ is an absolute integral invariant of $X$. This means

$$
X\lrcorner \mathrm{d} \alpha=0 .
$$

As an example (classical mechanics, harmonic oscillator with $\left.H=x^{2} / 2+y^{2} / 2\right)$, let

$$
\alpha=y \mathrm{~d} x-\left(x^{2} / 2+y^{2} / 2\right) \mathrm{d} t
$$

and

$$
Y=-x \partial / \partial y+y \partial / \partial x+\partial / \partial t .
$$

Then we find that $Y\lrcorner \mathrm{d} \alpha=0$, i.e. $\alpha$ is a relative integral invariant w.r.t. $Y$.

The trajectories of the vector field $Y$ will be invariant under a one-parameter group of transformation generated by a vector field $X$, if $X$ satisfies

$$
[X, Y]=f Y .
$$

Obviously, any vector parallel to $Y$ trivially satisfies this equation. The question as to how to obtain the form or the vector field $Y$ for a given $X$ has been discussed by the author [27-31]. The special case of Eq. (55) namely $[X, Y]=0$ is an integrability conditions. The geometrical meaning is that the (local) flows $\left(\Phi_{s}, \Psi_{t}\right)$ associated with $X$ and $Y$ commute, this means $\Phi_{s} \circ \Psi_{t}=\Psi_{t} \circ \Phi_{s}$. An illustrative example is given by the vector fields

$$
X=x \partial / \partial y-y \partial / \partial x
$$

and

$$
Y=x \partial / \partial x+y \partial / \partial y .
$$

In the present example we obtain the integrating factor $X^{1} Y^{2}-Y^{1} X^{2}=x^{2}+y^{2}$.

The relation between Eq. (47) and Eq. (55) can be seen by the following theorem: Theorem: Let $M=\mathbb{R}^{n}$ (or any open subset of $\mathbb{R}^{n}$ ) and $\omega=$ $\mathrm{d} x_{1} \wedge \cdots \wedge \mathrm{d} x_{n}$. Let $X$ and $Y$ be two vector fields such that $[X, Y]=f Y$. Then [31].

$$
\left.\left.L_{X}(Y\lrcorner \omega\right)=(f+\operatorname{div} X)(Y\lrcorner \omega\right)
$$

Finally, let us consider functions (although included in Eq. (48) as a special case, namely $\alpha$ is considered as a 0 -form). If $L_{X} f=0$, then we call $f$ an invariant w.r.t. $X$ (first integral of the dynamic system $\dot{x}=X(x)$ ). Now let $f(x)=0$ be an equation. Then $f$ is called a relative invariant w.r.t. $X$ if $L_{X} f=X f=0$ whether identically or by virtue of the equation $f=0$. In the former case the equation $f=c$ is invariant, and in the latter case not invariant, for all values of the constant $c$.

Equations (47), (55) have been studied a great deal in physics [1-22]. The author has applied these equations for investigating dynamic systems containing limit cycles and periodic orbits [27-31].

If some invariants are known, other invariants can be calculated. We have the following properties : Let $X$ be a vector field on $M$, and $\alpha, \beta$ invariant forms of $X$. Then $X\lrcorner \alpha, \mathrm{d} \alpha$, and $\alpha \wedge \beta$ are invariant forms of $X$. The proof can be found in Abraham [1].

Let us consider conformal invariance. $X\lrcorner \alpha$ and $\alpha \wedge \beta$ are conformal invariant forms of $X$, if $\alpha$ and $\beta$ are conformal invariants under $X$. To prove the first assertion we consider the identity (9). If $L_{X} \alpha=f \alpha$, then $\left.\left.L_{X}(X\lrcorner \alpha\right)=f(X\lrcorner \alpha\right)$. A further contraction with the vector field $X$ leads to nothing new. The reason is that for every vector field $X$ and every form $\alpha$ we get $X\lrcorner(X\lrcorner \alpha)=0$. To check the second assertion we apply the identity (6). If $L_{X} \alpha=f \alpha$ and $L_{X} \alpha=g \alpha$, then

$$
L_{X}(\alpha \wedge \beta)=(f+g)(\alpha \wedge \beta) .
$$

It should be mentioned that in general $\mathrm{d} \alpha$ is not a conformal invariant w.r.t. $X$, if $\alpha$ is conformal invariant w.r.t. $X$. To see this we apply the identity (8) and the relation $\mathrm{d}(f \alpha)=(\mathrm{d} f) \wedge \alpha+f \mathrm{~d} \alpha$. However, 
if $f$ is a constant, then $\mathrm{d} \alpha$ is a conformal invariant w.r.t. $X$. In order to treat partial differential equations within the geometrical approach the notion of invariance must be extended to include such cases as described above.

Let $X$ and $Y$ be two vector fields. Assuming $L_{X} \alpha=0$ and $L_{Y} \alpha=0$, we find that $L_{[X, Y]} \alpha=0$. Invoking identity (10) we can easily prove the statement. On the other hand, if we consider conformal invariance, i.e. $L_{X} \alpha=f \alpha$ and $L_{Y} \alpha=g \alpha$ then we cannot conclude that $L_{[X, Y]} \alpha=h \alpha$. For a special case the assertion holds, namely when both functions $f$ and $g$ are constants. This is due to the facts that $\mathrm{d}(X\lrcorner c \alpha)=c d(X\lrcorner \alpha)$ and

$$
(X\lrcorner d(c \alpha))=c(X\lrcorner(\mathrm{d} \alpha))
$$

where $c$ is a constant.

\section{Applications to Physies}

Now let us apply the developed tool to some problems in physics. Since we are studying problems in space-time, the vector field under investigation takes the form

$$
Y=\sum_{i=1}^{n} X^{i} \frac{\partial}{\partial x_{i}}+\frac{\partial}{\partial t}
$$

with the function $X^{i}(x, t)$. The above vector field is usually called the Eulerian (velocity) field. Throughout we investigate the case $n=3$. Sometimes we modify the vector field slightly according to $f Y$, where $f(x, t)$ is a smooth function. Now we can apply, via the Lie derivative, the vector field $Y$ to 0 -forms, 1-forms, 2-forms, 3-forms, and 4-forms. The problem will be considered from an opposite point of view. We require that $L_{Y}()=$.0 , where (.) stands for the form. Now we ask whether or not the resulting equations have a physical meaning. Consequently, what we have to do is to identify both the vector field $X$ and the form with physical quantities. It will be shown that this task can be done. Thus, the invariance requirement yields physical laws.

As useful abbreviations we introduce

$$
\Omega=\mathrm{d} x_{1} \wedge \mathrm{d} x_{2} \wedge \mathrm{d} x_{3} \wedge \mathrm{d} t
$$

and

$$
\omega=\mathrm{d} x_{1} \wedge \mathrm{d} x_{2} \wedge \mathrm{d} x_{3} .
$$

Hence, $\Omega$ is the volume element in space-time and $\omega$ the spatial volume element.
Consider first the vector field $f Y$ and the 4-form $\Omega$. Using Eq. (5) and Eq. (7) we find that

$$
\begin{aligned}
L_{f Y} \Omega=\left(\frac{\partial f}{\partial t}\right. & +X^{1} \frac{\partial f}{\partial x_{1}}+X^{2} \frac{\partial f}{\partial x_{2}} \\
& \left.+X^{3} \frac{\partial f}{\partial x_{3}}+f \operatorname{div} X\right) \Omega .
\end{aligned}
$$

Demanding that $L_{f Y} \Omega=0$, then, after a little algebraic manipulation, we obtain

$$
\frac{\partial f}{\partial t}+\sum_{i=1}^{3} \frac{\partial\left(f X^{i}\right)}{\partial x_{i}}=0
$$

because $\Omega$ is a basic element. If we identify the quantity $f$ with the mass density and $X$ with the velocity vector field $\left(X^{i}=\mathrm{d} x_{i} / \mathrm{d} t\right)$, then the above equation describes nothing more than the wellknown continuity equation in local (differential) form. Obviously, we get the same result when we consider the field $Y$ and the form $f \Omega$.

The energy balance equation can readily be obtained by slightly modifying the example given above. Now we start with the vector field $(f g) Y+A$, where

$$
A=A^{1} \frac{\partial}{\partial x_{1}}+A^{2} \frac{\partial}{\partial x_{2}}+A^{3} \frac{\partial}{\partial x_{3}}
$$

with the function $A^{i}(x, t)$. Both $f$ and $g$ are smooth function of $x$ and $t$. We require that

$$
L_{(f g Y+A)} \Omega=(f p) \Omega .
$$

This means we consider conformal invariance. By straightforward calculation we find that

$$
\frac{\partial(f g)}{\partial t}+\sum_{i=1}^{3} \frac{\partial\left(f g X^{i}\right)}{\partial x_{i}}+\sum_{i=1}^{3} \frac{\partial A^{i}}{\partial x_{i}}=f p ;
$$

$f$ is the mass density, $g$ the total energy per unit mass, $A$ represents the heat flux density, and $f p$ the internal heat generation rate per unit volume.

Requiring that $L_{Y} f \omega=0$, then we also obtain the continuity equation, however an additional condition appears. We have

$$
\begin{aligned}
L_{Y} f \omega= & \left(\frac{\partial f}{\partial t}+\sum_{i=1}^{3} \frac{\partial\left(f X^{i}\right)}{\partial x_{i}}\right) \omega \\
& +f\left(\frac{\partial X^{1}}{\partial t} \mathrm{~d} x_{2} \wedge \mathrm{d} x_{3} \wedge \mathrm{d} t+\frac{\partial X^{2}}{\partial t}\right. \\
& \left.\cdot d x_{3} \wedge \mathrm{d} x_{1} \wedge \mathrm{d} t+\frac{\partial X^{3}}{\partial t} \mathrm{~d} x_{1} \wedge \mathrm{d} x_{2} \wedge \mathrm{d} t\right) .
\end{aligned}
$$


Besides the continuity equation, we get the conditions

$$
\frac{\partial X^{1}}{\partial t}=\frac{\partial X^{2}}{\partial t}=\frac{\partial X^{3}}{\partial t}=0
$$

Equation (65) tells us that the flow is steady.

Before proceeding further it will be necessary to explain the connection between the Lie derivative and what in hydrodynamics is called the substantial or material derivative. Let $X=\left(X^{1}, X^{2}, X^{3}\right)$ be a velocity field. Then the substantial derivative of $X$, denoted by $D X \mid D t$, is defined as

$$
\begin{aligned}
\frac{D X}{D t}=\frac{\partial X}{\partial t} & +(X \cdot \operatorname{grad}) X=\frac{\partial X}{\partial t} \\
& +\operatorname{grad}\left((X)^{2} / 2\right)-X \times \operatorname{curl} X
\end{aligned}
$$

where $(X)^{2}=\left(X^{1}\right)^{2}+\left(X^{2}\right)^{2}+\left(X^{3}\right)^{2}$. In cartesian coordinates let $\alpha=X^{1} \mathrm{~d} x_{1}+X^{2} \mathrm{~d} x_{2}+X^{3} \mathrm{~d} x_{3}$. Then it follows that

$$
\begin{aligned}
L_{Y} \alpha= & \sum_{j=1}^{3}\left(\frac{\partial X_{j}}{\partial t}+\sum_{i=1}^{3} X^{i} \frac{\partial X_{j}}{\partial x_{i}}\right. \\
& \left.+\sum_{i=1}^{3} X^{i} \frac{\partial X^{i}}{\partial x_{j}}\right) \mathrm{d} x_{j}+\left(\sum_{i=1}^{3} X^{i} \frac{\partial X^{i}}{\partial t}\right) \mathrm{d} t .
\end{aligned}
$$

Consequently, the expression $L_{Y} \alpha-\mathrm{d}\left((X)^{2} / 2\right)$ is equal to the expression given by Equation (66). Now we are able to establish the Eulerian equation (or particular forms of it) in the language of Lie derivative and exterior derivative. For example, the equation

$$
L_{Y} \alpha=\mathrm{d}\left((X)^{2} / 2\right)-f^{-1} \mathrm{~d} p
$$

yields

$$
\begin{gathered}
\partial X / \partial t+\operatorname{grad}\left((X)^{2} / 2\right)-X \times \operatorname{curl} X \\
=-f^{-1} \operatorname{grad} p
\end{gathered}
$$

$f$ is the mass density, and $p$ denotes the pressure. Thus Eq. (69) describes the Eulerian equation.

In the present example we have mainly considered cartesian coordinates, but we emphasize that the operators $d($.$) and L_{X}($.$) are coordinate free.$

Finally, let us discuss some problems concerning the notions ,grad", ,curl" and ,div" in spacetime. In $\mathbb{R}^{3}$ with coordinates $x, y, z$ we have the following properties: Let $\Phi=\Phi(x, y, z)$. The equation $\mathrm{d} \Phi=0$ is equivalent to $\operatorname{grad} \Phi=0$, because $\mathrm{d} x, \mathrm{~d} y$, and $\mathrm{d} z$ are basic forms. Let

$$
\alpha=E_{1} \mathrm{~d} x+E_{2} \mathrm{~d} y+E_{3} \mathrm{~d} z .
$$

Then the requirement $\mathrm{d} \alpha=0$ is equivalent to curl $E=0$ with the vector $E=\left(E_{1}, E_{2}, E_{3}\right)$, because $\mathrm{d} x \wedge \mathrm{d} y, \mathrm{~d} y \wedge \mathrm{d} z$, and $\mathrm{d} z \wedge \mathrm{d} x$ are basic forms. Now let $\beta=B_{12} \mathrm{~d} x \wedge \mathrm{d} y+B_{23} \mathrm{~d} y \wedge \mathrm{d} z+B_{31} \mathrm{~d} z \wedge \mathrm{d} x$. It follows that $\mathrm{d} \beta=0$ is equivalent to $\operatorname{div} B=0$ with the vector $B=\left(B_{23}, B_{31}, B_{12}\right)$. In space-time the situation becomes more complicated. In space-time we obtain physical laws for appropriate forms. The continuity equation can be expressed as follows. Let

$$
\begin{aligned}
\alpha=f(\mathrm{~d} x \wedge \mathrm{d} y \wedge \mathrm{d} z & -X^{1} \mathrm{~d} y \wedge \mathrm{d} z \wedge \mathrm{d} t \\
& -X^{2} \mathrm{~d} z \wedge \mathrm{d} x \wedge \mathrm{d} t \\
& \left.-X^{3} \mathrm{~d} x \wedge \mathrm{d} y \wedge \mathrm{d} t\right) .
\end{aligned}
$$

Applying the rule $\mathrm{d}(g \lambda)=(\mathrm{d} g) \wedge \lambda+g \mathrm{~d} \lambda$, we find that the requirement $d \alpha=0$ leads to the continuity equation. $f$ is the mass density and $X$ the velocity field. Consider next the 2 -form

$$
\begin{aligned}
\beta=B_{23} \mathrm{~d} y \wedge \mathrm{d} z & +B_{12} \mathrm{~d} x \wedge \mathrm{d} y+B_{31} \mathrm{~d} z \wedge \mathrm{d} x \\
& +E_{1} \mathrm{~d} x \wedge \mathrm{d} t+E_{2} \mathrm{~d} y \wedge \mathrm{d} t \\
& +E_{3} \mathrm{~d} z \wedge \mathrm{d} t .
\end{aligned}
$$

The equation $\mathrm{d} \beta=0$ yields $\operatorname{curl} E=-\partial B / \partial t$ and $\operatorname{div} B=0$. The first equation is Faraday's law of induction and the second one the nonexistence of true magnetism. Finally, let us consider the 1-form $\gamma=X^{1} \mathrm{~d} x+X^{2} \mathrm{~d} y+X^{3} \mathrm{~d} z-\left((X)^{2} / 2\right) \mathrm{d} t-p \mathrm{~d} t$, where $(X)^{2}=\left(X^{1}\right)^{2}+\left(X^{2}\right)^{2}+\left(X^{3}\right)^{2}$. The condition $\mathrm{d} \gamma=0$ leads to the equation $\operatorname{curl} X=0$ and $\partial X / \partial t+\operatorname{grad}\left((X)^{2} / 2\right)=-\operatorname{grad} p$. Thus we find a particular form of the Eulerian equations, i.e. curl $X$ vanishes. $p$ denotes the pressure. Obviously, the described approach can be extended by considering the condition $\mathrm{d} \alpha=\beta$ instead of $\mathrm{d} \alpha=0$. Thus $\beta$ is an $r$-form and $\alpha$ an $(r-1)$-form.

\section{Conclusion}

To sum up, we have shown that the concept of Lie derivative is a powerful tool for describing physical laws. In particular, the balance equations can be obtained rigorously. The given approach can also be applied to differential conservation laws in the theory of relativity. However, some quantities in this field cannot be represented as a differential form. Here we must use Eqs. (42) through (46).

Since the physicist is not familiar in general with the rules of manipulation of the Lie derivative a short review about the properties of the Lie derivative has been given. Throughout we have studied local forms of the derived equations. In most cases the euclidian space has been the underly. ing Riemannian manifold. 
To extend the present theory it is necessary to include integration on manifolds and in particular the theorem of Stokes [32]. With this tool we are able to introduce quantization.

In the present paper we have considered the concept of invariance, conformal invariance, integral invariance, and relative integral invariance. Relative integral invariance plays the central role in classical mechanics. The concepts of invariance and conformal invariance are important for deriving balance equations. To treat partial differential equations (linear and non-linear) within the present approach the concept of invariance must be extended. The partial differential equation is cast into a system of partial differential equations of first order and then into an equivalent system of differential forms. The equivalent system of differential forms is a differential ideal [33]. An example of a differential ideal is given by Equation (51).

In physics it is known that via the concept of variation (what in mathematics is called the Fréchet derivative of certain mappings between Banach spaces) physical laws can be obtained (as

[1] R. Abraham, Foundations of Mechanics, Benjamin, New York 1967.

[2] R. Hermann, Differential Geometry and the Calculus of Variations, Academic Press, New York 1968.

[3] R. Hermann, Vector Bundles in Mathematical Physics Volume II, Benjamin, New York 1970.

[4] W. Slebodzinski, Formes Extérieures et leurs Applications, Vols. I and II, Pánstwowe Wydawnictwo Naukowe 1954, 1963.

[5] Y. Choquet-Bruhat, Géométrie Différentielle et Systèmes Extérieur, Dunod, Paris 1968.

[6] C. Godbillon, Géométrie Differentielle et Mécanique Analytique, Hermann, Paris 1969.

[7] J. Komorowski, Rep. Math. Phys. 1, 105 (1970).

[8] R. M. Kiehn, J. Math. Phys. 15, 9 (1974).

[9] R. M. Kiehn, J. Math. Phys. 16, 1032 (1975).

[10] M. Andrié, J. Math. Phys. 17, 394 (1976).

[11] R. M. Kiehn, Int. J. Engng. Sci. 13, 941 (1975).

[12] D. G. Ebin and J. Marsden, Ann. Math. 92, 102 (1970).

[13] T. J. R. Hughes and J. Marsden, Rep. Math. Phys. 12, 35 (1977).

[14] G. A. Deschamps, Exterior Differential Forms in Mathematics. Applied to Physics, Editor R. Roubine, Springer-Verlag, Berlin 1970.

[15] C. von Westenholz, Found. Physics 7, 529 (1977).

[16] C. von Westenholz, Int. J. Theor. Phys. 10, 391 (1974).

[17] E. Grgin and A. Petersen, Int. J. Theor. Phys. 6, 325 (1972).

[18] F. Mansouri, J. Math. Phys. 18, 52 (1972).

[19] N. Hurt, Int. J. Theor. Phys. 7, 263 (1973). a typical example the Lagrange equation). Both concepts are connected [33].

Finally we mention a point of view which has been overlooked so far. Moreover, in some books $[14,34]$ this point of view has not been taken into account at all. In Section 3 we have introduced mass density as the three form

$$
f(x, t) \mathrm{d} x_{1} \wedge \mathrm{d} x_{2} \wedge \mathrm{d} x_{3} .
$$

Because of certain transformation properties the density must be considered as a current [35] (in the present case as a 0-current). However, the considerations given above remain true, because such questions don't play any role in the present case. A current is a linear form defined via an integral over the form $\alpha \wedge \Phi$ where $\Phi$ is a so-called $k$-test-form and $\alpha$ a $(n-k)$-form. For many purpose one can identify the form $\alpha$ and its associated current $T_{\alpha}$ (for details see [35]).

\section{Acknowledgements}

Thanks are due to Dr. W. Barry for a critical reading of the manuscript.

[20] S. Manoff, Acta Phys. Aust. 48, 41 (1977).

[21] M. L. Woolley, J. Phys. A 10, 2107 (1977).

[22] M. Kovacich, Foundations of a General Relativistic Statistical Mechanics for Interacting Particles and a Derivation of the General Relativistic Boltzmann Equation, University of California, Irvine, Preprint 1977.

[23] B. K. Harrison and F. B. Estabrook, J. Math. Phys. 12, 653 (1971).

[24] C. P. Boyer and E. G. Kalnins, J. Math. Phys. 18, 1032 (1977).

[25] F. B. Estabrook and H. D. Wahlquist, SIAM Review 17, 201 (1975).

[26] J. Dieudonné, Éléments d'Analyse 4 (p. 15), GauthierVillars, Paris 1971.

[27] W.-H. Steeb, Phys. Lett. 62 A, 211 (1977).

[28] W.-H. Steeb, Proc. 13th IUPAP Int. Conf. on Stat. Physics, Haifa, 1977 (in press).

[29] W.-H. Steeb, Lett. Math. Phys. 2, 171 (1977).

[30] W.-H. Steeb, J. Phys. A 10, L221 (1977).

[31] W.-H. Steeb, Int. J. Theor. Phys. (1978) (in press).

[32] M. Spivak, Calculus on Manifolds, Benjamin, New York 1965.

[33] R. Hermann, Geometry, Physics and Systems, Dekker, New York 1973.

[34] H. Flanders, Differential forms, Academic Press, New York 1963.

[35] J. Dieudonné, Éléments d'Analyse 3 (p. 234), Gauthier Villars, Paris 1974.

Nachdruck - auch auszugsweise - nur mit schriftlicher Genehmigung des Verlages gestattet

Verantwortlich für den Inhalt: A. KLem

Satz und Druck: Konrad Triltsch, Würzburg 